\title{
Clasificación de las secciones rectangulares de hormigón armado por los criterios de cálculo: compresión, interpolaciones y flexión.
}

(Instrucción EH-73)

JULIAN NAVARRO GUTIERREZ,

Dr. Arquitecto

$432 \cdot 3$

\begin{abstract}
Sinopsis En cualquier caso de cálculo de una sección rectangular de hormigón armado, a partir de sus datos y antes En cualquier caso de cálculo de una sección rectangular de hormigón armado, a partir de sus datos antes de hacer el correspondiente cálculo, es posible determinar fácilmente dos números que, adoptados como
coordenadas, determinan un punto. La situación de este punto en su plano de representación indica el sistema ce ecuaciones que ha de ser utilizado para el cálculo de la sección. En los casos de compatibilidad hay cuatro posibilidades: compresión (simple o compuesta), compresión interpolada, flexión interpolada y flexíón (simple o compuesta). Corresponde a cada una de estas posibilidades un determinado recinto, finito o infinito, en el plano de representación. Para obtener los contornos de estos recintos es necesario previamente elegir el valor del parámetro $\varrho=$ coeficiente de recubrimiento de las armaduras.

$\varrho=$ coeficiente de recubrimiento de las armaduras;

$\mathrm{s}_{\mathrm{c}}=$ coeficiente de rotura del hormigón.

Casi todos los casos corrientes de cálculo se agrupan en:

1. Determinación de secciones en las que el ancho b es dato y el canto $d$ es incógnita.

II. Determinación de secciones en las que b y d son datos.

A cada grupo corresponde un determinado gráfico, si previamente se ha establecido el valor de $\varrho$.
\end{abstract}

\section{PRELIMINAR}

Los parámetros simples geométricos de una sección rectangular de hormigón armado, como la representada en la figura 1, son:

$$
b, d, d^{\prime}, A_{s}, A_{s}^{\prime}, e, y
$$

Los parámetros simples mecánicos de la misma sección son:

$$
f_{c}, f_{s}, N
$$

Su significación queda determinada en la indicada figura 1 y con las siguientes equivalencias:

$e=$ distancia del punto de aplicación de $N$ al centro de $A_{s}$.

$y=$ profundidad de la zona del hormigón comprimido.

$f_{c}=$ coeficiente de rotura del hormigón.

$f_{s}=$ coeficiente de fluencia del acero.

En total son diez parámetros simples.

Mediante ellos se definen los siguientes parámetros compuestos:

$\varrho=d^{\prime}: d=$ coeficiente de recubrimiento de las armaduras

$U_{c}=b \times d \times t_{c}=$ capacidad mecánica de la sección de hormigón.

$U^{\prime}{ }_{s}=A_{s}^{\prime} \times f_{s}=$ capacidad mecánica de la armadura $A^{\prime}{ }_{s}$.

$U_{s}=A_{s} \times f_{s}=$ capacidad mecánica de la armadura $A_{s}$.

$r=U_{s}^{\prime}: U_{c}=$ cuantía en armadura $A_{s}^{\prime}$.

$q=y: d=$ profundidad relativa de la zona comprimida.

$c=$ factor en $c \times f_{c}$, que es el valor unitario de compresión en el hormigón.

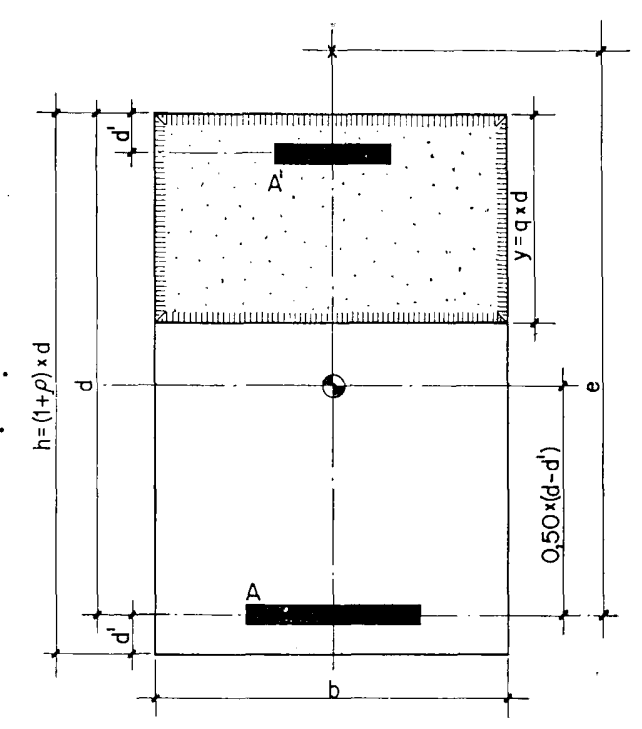

Fig. 1.

$h=d \times(1+\varrho)=$ altura de la sección rectangular de hormigón.

Sección rectangular de hormigón armado. Parámetros geométricos. 
El conjunto de los diez parámetros simples, u otro conjunto de parámetros simples y com puestos equivalente al primero, se designa con la locución «sección».

Cuando la armadura $A_{s}$ se halla fuera de la zona del hormigón comprimido la expresión del momento tope de la sección es:

$$
\mu_{1}=0,35 \times d \times U_{c}+U_{s}^{\prime} \times\left(d-d^{\prime}\right)=[0,35+r \times(1-\varrho)] \times d \times U_{c}
$$

Cuando ambas armaduras $A_{s}, A_{s}^{\prime}$ se hallan en la zona comprimida, la expresión de dicho momento tope es:

$$
\mu_{2}=0,35 \times U_{c} \times\left(1-\varrho^{2}\right)+U_{s}^{\prime} \times\left(d-d^{\prime}\right)=\left[0,35 \times\left(1-\varrho^{2}\right)+r \times(1-\varrho)\right] \times d \times U_{c}
$$

Designando con $\mu$ uno $\mathrm{u}$ otro de ambos momentos tope, la primera clasificación de las secciones se establece mediante su relación con el momento $N \times e$ de las acciones exteriores.

Son incompatibles las secciones en que se verifica $N \times e>\mu$. Las restantes secciones son compatibles, y se dividen en secciones tope, cuando es $N \times e=\mu$, y secciones de canto dado, cuando es $N \times e<\mu$.

La expresión general del momento $N \times e$ es

$$
N \times e=[q \times c \times(1-0,50 \times q)+r \times(1-\varrho)] \times d \times U_{c}
$$

tanto si es $q<1$ como si es $q>1$. En todo caso es $q \leqslant 1+\varrho$.

Las secciones tope se pueden clasificar en las siguientes clases:

1. Compresión, cuya característica general es

$$
N \times e=\left[0,35 \times\left(1-\varrho^{2}\right)+r \times(1-\varrho)\right] \times d \times U_{c}
$$

con las dos subclases siguientes:

1.1. Compresión compuesta, con los parámetros $q=1+\varrho, c=0,70$.

1.2. Compresión interpolada, con las condiciones

$$
c \times q \times(1-0,50 \times q)=0,35 \times\left(1-\varrho^{2}\right) \quad ; \quad U_{s}=0
$$

2. Flexión, cuya característica general es

$$
N \times e=[0,35+r \times(1-\varrho)] \times d \times U_{c}
$$

y con las dos subclases siguientes:

2.1. Flexión interpolada, con las condiciones

$$
c \times q \times(1-0,50 \times q)=0,35 \quad ; \quad U_{s}=0
$$

2.2. Flexión compuesta, con los parámetros

$$
q=0,45 ; c=1
$$

A su vez, las secciones de canto dado se pueden, análogamente, clasificar del siguiente modo:

3. Compresión, cuya característica general es

$$
\frac{N \times e}{d \times U_{c}}-r \times(1-\varrho)<0,35 \times\left(1-\varrho^{2}\right)
$$

con las dos subclases siguientes:

3.1. Compresión compuesta, con los parámetros

$$
q=1+\varrho \quad ; \quad c<0,70
$$


3.2. Compresión interpolada, con las condiciones

$$
c \times q \times(1-0,50 \times q)<0,35 \times\left(1-\varrho^{2}\right) ; \quad U_{s}=0
$$

4. Flexión, cuya característica general es

$$
\frac{N \times e}{d \times U_{c}}-r \times(1-\varrho)<0,35
$$

con las dos subclases siguientes:

4.1. Flexión interpolada, con las condiciones

$$
c \times q \times(1-0,50 \times q)<0,35 ; U_{s}=0
$$

4.2. Flexión compuesta, con los parámetros

$$
q<0,45 ; c=1
$$

Casi todos los casos de cálculo de secciones se hallan en la práctica incluidos en alguno de los 8 casos anteriores.

Es muy curioso y muy útil el hecho de que sea posible, en cada uno de los 8 casos indicados, antes de realizar cada cálculo, obtener fácilmente las coordenadas de un punto, que parece apropiado llamar "punto selector», y que los puntos selectores pertenecientes a cada proceso de cálculo llenen un recinto plano y distinto para cada proceso. Como dichos recintos pueden quedar determinados de antemano, la simple ubicación del punto selector indica cuál es el proceso adecuado de cálculo, sin tanteos ni incertidumbres (salvo una pequeña faja fronteriza entre las interpolaciones de las secciones tope).

A los 4 casos de las secciones tope, y a cada valor particular de $\varrho$, corresponde una figura plana, compuesta por los 4 indicados recintos. Los recintos de las 2 interpolaciones presentan un pequeño solape, que no produce confusión, como se verá más adelante. Véase la figura 2, que corresponde a $\varrho=0,10$.

Análogamente, a los 4 casos de las secciones de canto dado, y a cada valor de $\varrho$, corresponde una figura plana con los recintos respectivos. En la figura 3, que acompaña a este texto se han dibujado las separaciones o contornos para $\varrho=0,10$.

La expresión general de $N \times e$ y la expresión general

$$
N=(c \times q+r) \times U_{c} \pm U_{s}
$$

son las dos ecuaciones del equilibrio en la rotura de una sección rectangular, y de ellas se deducen todas las correspondientes a los casos particulares.

A continuación se estudian las ecuaciones y la distribución de los puntos selectores correspondientes a dichos casos particulares.

\section{SECCIONES TOPE}

Las coordenadas del punto selector para las secciones tope son:

$$
Z=\frac{N}{e \times b \times f_{c}} \quad ; \quad r=\frac{U_{s}^{\prime}}{U_{c}}
$$

cuyos respectivos intervalos de variabilidad son $(0,+\infty),(0,0,50)$, respectivamente. Dichas coordenadas se suponen colocadas en un sistema cartesiano ortogonal (fig. 2).

1. Compresión. La expresión [1.m] tiene que ser igual a

por consiguiente:

$$
N \times e=\left[0,35 \times\left(1-\varrho^{2}\right)+r \times(1-\varrho)\right] \times d \times U_{c}
$$

$$
d=\sqrt{\frac{N \times e}{b \times f_{c} \times\left[0,35 \times\left(1-\varrho^{2}\right)+r \times(1-\varrho)\right]}} \quad ; \quad U_{c}=b \times d \times f_{c}
$$




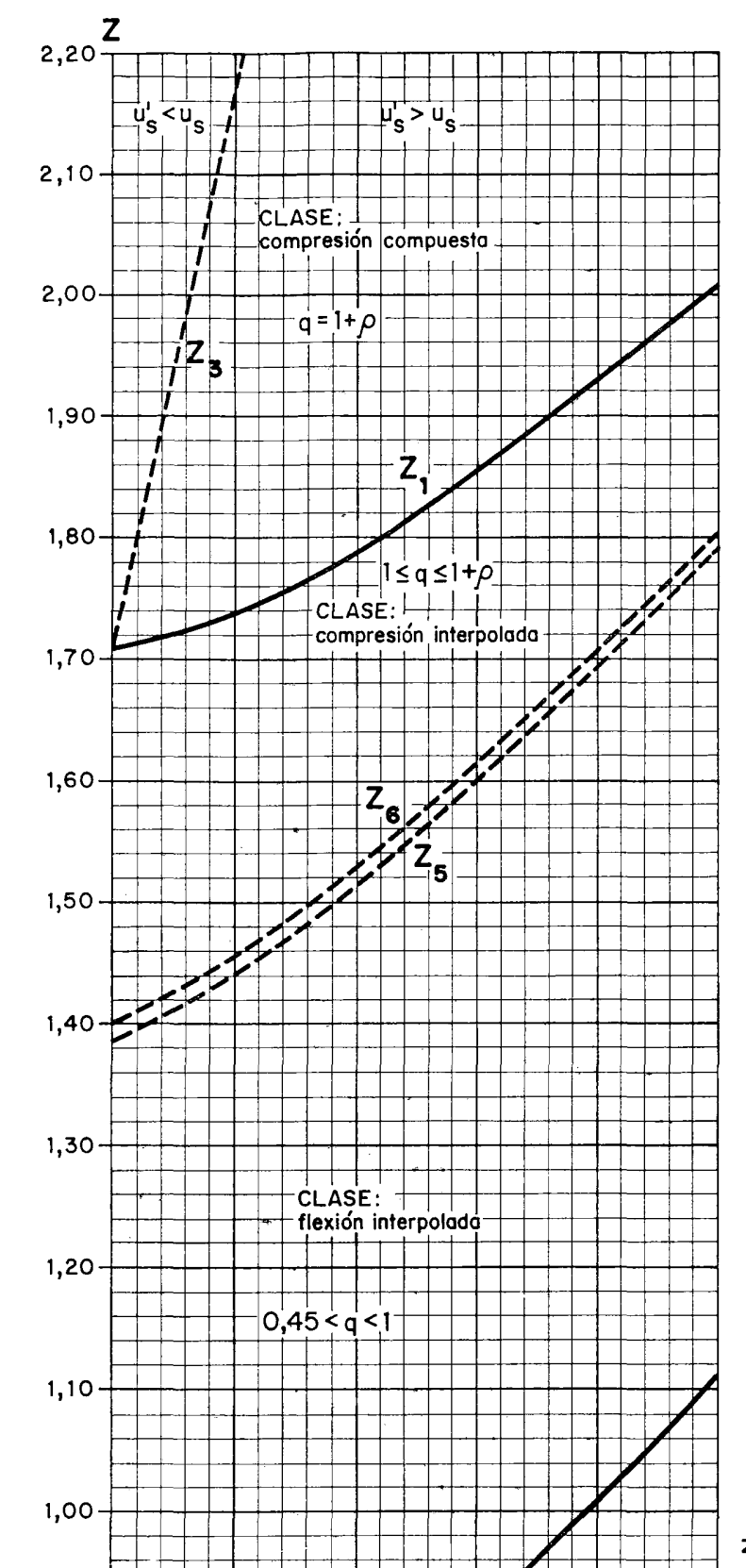

Figura 2

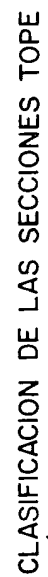

Figura 3

을으 1,00 Q

岁"

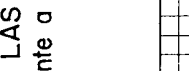

㟧 $\frac{\mathbf{\Phi}}{\mathrm{g}}$

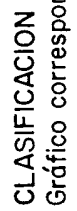

0,90

$+-11+1$

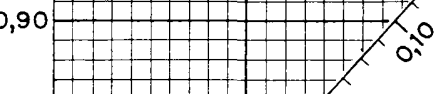

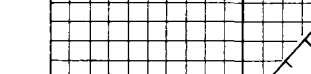
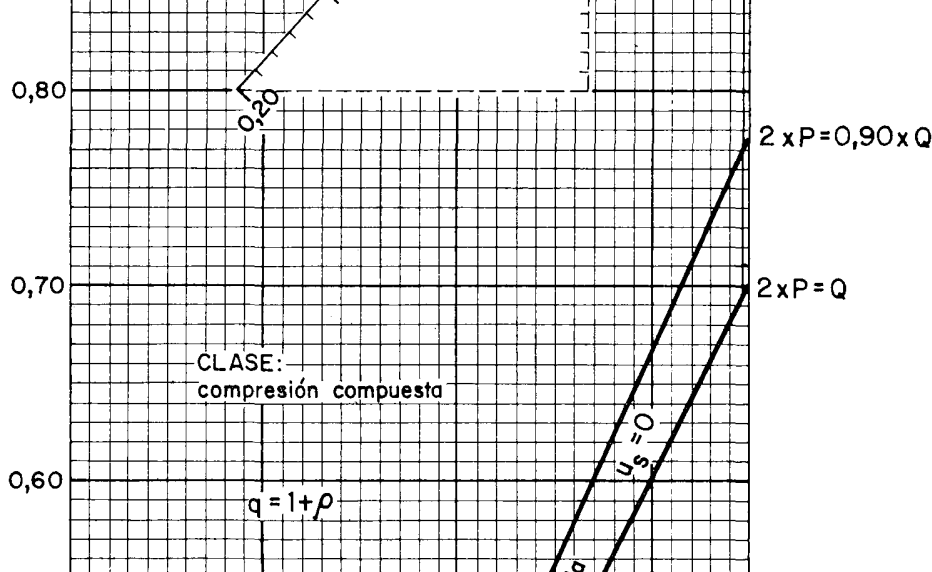
$2 \times P=Q$

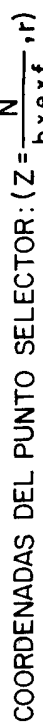
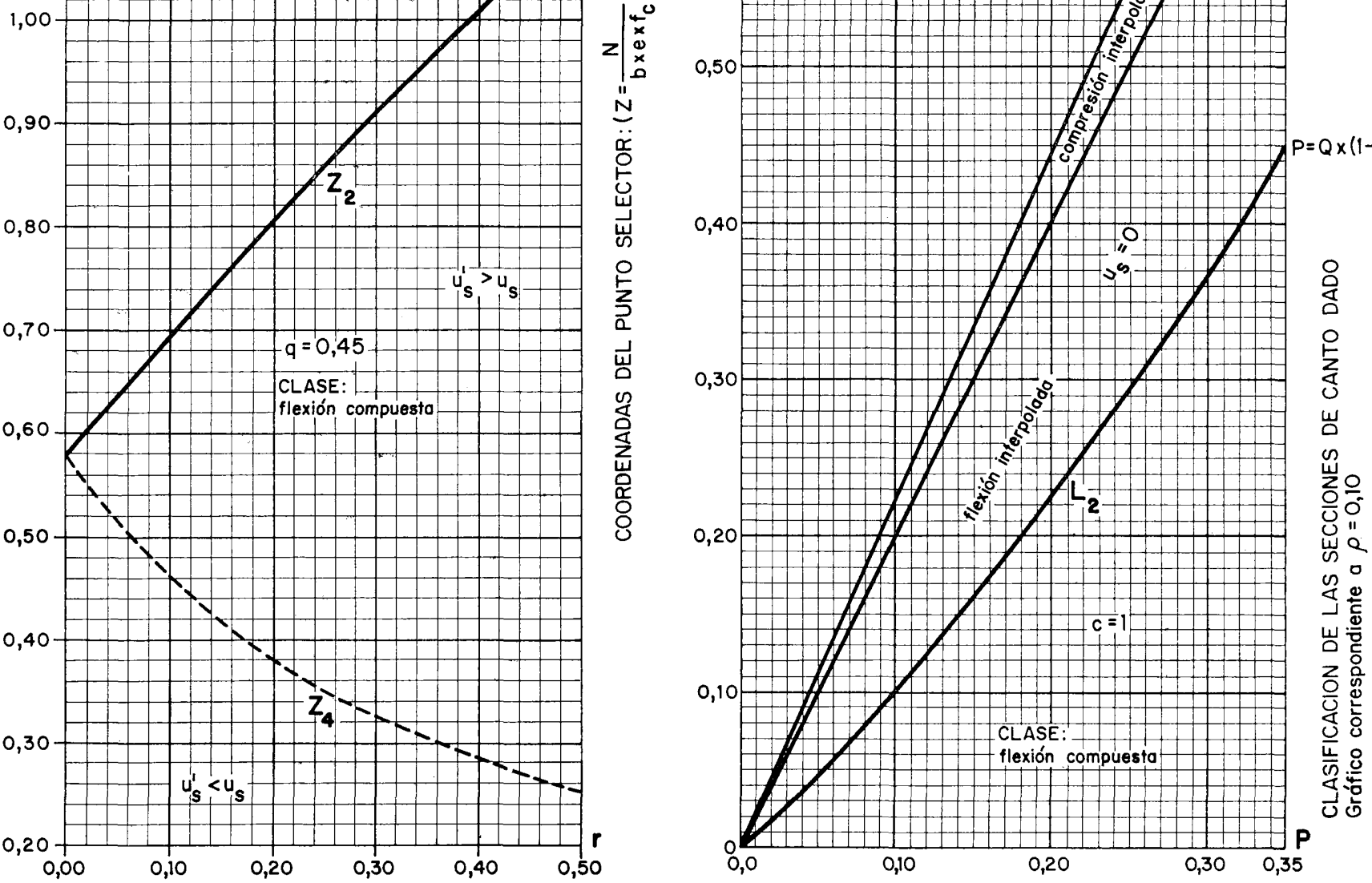
1.1. Compresión compuesta. Como han de ser $q=1+\varrho, c=0,70$, la expresión [1.r] se transforma en

$$
N=[0,70 \times(1+\varrho)+r] \times U_{c}+U_{s}
$$

quedando

$$
U_{s}=N-[0,70 \times(1+\varrho)+r] \times U_{c} \quad ; \quad U^{\prime}=r \times U_{c}
$$

Estas fórmulas no tienen validez cuando resulte $U_{s}<0$.

El límite de validez corresponde a $U_{s}=0$, o sea, a

$$
N^{2}=[0,70 \times(1+\varrho)+r]^{2} \times b^{2} \times f_{c}^{2} \times \frac{N \times e}{b \times f_{c} \times\left[0,35 \times\left(1-\varrho^{2}\right)+r \times(1-\varrho)\right]}
$$

y esta fórmula, una vez simplificada, es

$$
Z_{1}=\frac{[0,70 \times(1+\varrho)+r]^{2}}{0,35 \times\left(1-\varrho^{2}\right)+r \times(1-\varrho)}
$$

y corresponde en el campo de los puntos selectores a la línea $Z_{1}$.

1.2. Compresión interpolada. Son $U_{s}=0 ; U_{s}^{\prime}=r \times U_{c}$.

Los parámetros $q$ y $c$ se deducen de

$$
c \times q \times(1-0,50 \times q)=0,35 \times\left(1-\varrho^{2}\right) \quad ; \quad N=(c \times q+r) \times U_{c}
$$

con los resultados siguientes:

$$
q=2-\frac{0,70 \times\left(1-\varrho^{2}\right) \times U_{c}}{N-r \times U_{c}} \quad ; \quad c=\left(\frac{N}{U_{c}}-r\right): q
$$

Los parámetros $c$ y $q$ no intervienen en la composición material de la sección, pero son imprescindibles para la comprobación de su cálculo.

Si en la ecuación $c \times q \times(1-0,50 \times q)=0,35 \times\left(1-\varrho^{2}\right)$ se hace sucesivamente $q=$ $=1+\varrho, q=1$ resultan, respectivamente, $c=0,70, c=0,70 \times\left(1-\varrho^{2}\right)$.

Los dos valores correspondientes $q=1+\varrho, c=0,70$, colocados en la expresión de $N$ dan

$$
N=[0,70 \times(1+\varrho)+r] \times U_{c}
$$

que es la misma fórmula obtenida para la línea $L_{1}$.

Los dos valores correspondientes $q=1, c=0,70 \times\left(1-\varrho^{2}\right)$, colocados en la expresión de $N$ dan

$$
N=\left[0,70 \times\left(1-\varrho^{2}\right)+r\right] \times U_{c}
$$

esta fórmula es equivalente a

$$
N^{2}=\left[0,70 \times\left(1-\varrho^{2}\right)+r\right]^{2} \times b^{2} \times f_{c}^{2} \times \frac{N \times e}{b \times f_{c} \times\left[0,35 \times\left(1-\varrho^{2}\right)+r \times(1-\varrho)\right]}
$$

que, una vez simplificada, es

$$
Z_{5}=\frac{\left[0,70 \times\left(1-\varrho^{2}\right)+r\right]^{2}}{0,35 \times\left(1-\varrho^{2}\right)+r \times(1-\varrho)}
$$

Esta fórmula, con $\varrho=0,10$, corresponde a la línea $Z_{5}$ de la figura 2 .

2. Flexión. La expresión [1.m] tiene que ser igual a

$$
N \times e=[0,35+r \times(1-\varrho)] \times d \times U_{c}
$$

por consiguiente,

$$
d=\frac{N \times e}{b \times f_{c} \times[0,35+r \times(1-\varrho)]} \quad ; \quad U_{c}=b \times d \times f_{c}
$$


2.1. Flexión interpolada. Son $U_{s}=0, U_{s}^{\prime}=r \times U_{c}$.

Los parámetros $q$ y $c$ se deducen de

$$
c \times q \times(1-0,50 \times q)=0,35 ; N=(c \times q+r) \times U_{c}
$$

con los resultados siguientes:

$$
q=2-\frac{0,70 \times U_{c}}{N-r \times U_{c}} \quad ; \quad c=\left(\frac{N}{U_{c}}-r\right): q
$$

A los valores $c=0,70, c=1$ corresponden, respectivamente, $q=1, q=0,45$, en la primera ecuación.

Los dos valores correspondientes, $c=0,70, q=1$, colocados en la segunda fórmula, dan $N=(0,70+r) \times U_{c}$, o sea,

$$
N^{2}=(0,70+r)^{2} \times b^{2} \times f_{c}^{2} \times \frac{N \times e}{b \times f_{c} \times[0,35+r \times(1-\varrho)]}
$$

o sea,

$$
Z_{6}=\frac{(0,70+r)^{2}}{0,35+r \times(1-\varrho)}
$$

Los dos valores correspondientes $c=1, q=0,45$, colocados en la misma segunda fórmula, dan: $N=(0,45+r) \times U_{c}$, o sea,

$$
N^{2}=(0,45+r)^{2} \times b^{2} \times f_{c}^{2} \times \frac{N \times e}{b \times f_{c} \times[0,35+r \times(1-\varrho)]}
$$

o sea,

$$
Z_{2}=\frac{(0,45+r)^{2}}{0,35+r \times(1-\varrho)}
$$

2.2. Flexión compuesta. Como han de ser $q=0,45, c=1$, la expresión [1.r] se transforma en

$$
N=(0,45+r) \times U_{c}-U_{s}
$$

quedando

$$
U_{s}=(0,45+r) \times U_{c}-N \quad ; \quad U_{s}{ }^{\prime}=r \times U_{c}
$$

Estas fórmulas no tienen validez cuando resulte $U_{s}<0$.

El límite de validez corresponde a $U_{s}=0$, o sea, a $N=(0,45+r) \times U_{c}$, y ésta es la fórmula que ha determinado la línea $Z_{2}$ en el párrafo precedente.

En resumen (véase la fig. 2 de $\varrho=0,10$ ), el campo de variabilidad del punto selector $(Z, r)$ queda dividido en varios recintos por las líneas $Z_{1}, Z_{2}, Z_{5}, Z_{6}$.

- Compresión compuesta. Todos los puntos situados por encima de la línea $Z_{1}$ corresponden a «compresión compuesta». En todos ellos se verifica $U_{s}>0$. En particular, hay puntos selectores en los que se verifica $U_{s}^{\prime}=U_{s}$. Cuando esto ocurre, la situación de la sección es de Compresión simple. En esta situación se verifica $N=[0,70 \times(1+\varrho)+2 \times r] \times$ $\times U_{c}, \mathrm{y}$ mediante el mismo proceso operativo repetido anteriormente, se determina la expresión

$$
Z_{3}=\frac{[0,70 \times(1+\varrho)+2 \times r]^{2}}{0,35 \times\left(1-\varrho^{2}\right)+r \times(1-\varrho)}=\frac{0,35 \times(1+\varrho)+r}{0,25 \times(1-\varrho)}
$$

que está representada en la figura 2 por la línea recta $Z_{3}$.

- Compresión interpolada. Los puntos selectores situados entre las líneas $Z_{1}$ y $Z_{5}$ corres. ponden a compresión interpolada. Los puntos situados en la línea $Z_{1}$ son indistintamente de compresión compuesta o de compresión interpolada. 
- Flexión interpolada. Los puntos situados entre las líneas $Z_{6}$ y $Z_{2}$ en el campo de variabilidad de $(Z, r)$ corresponden a secciones en flexión interpolada.

El pequeño campo de puntos situados entre las líneas $Z_{5}, Z_{6}$ pertenece indistintamente a compresión o a flexión interpoladas. Los resultados de ambas atribuciones son cuantitativamente muy próximos.

- Flexión compuesta. Corresponde a todos los puntos selectores situados por debajo de la línea $Z_{2}$, hasta el eje de las $r$. En los puntos de este eje es $Z=0$, y, por consiguiente, corresponden a las secciones en situación de flexión simple. Los puntos de la línea $Z_{2}$ son indistintamente de flexión interpolada o compuesta. En este recinto hay que destacar los puntos correspondiente a las secciones con armaduras simétricas. Cuando en una sección tope son de igual valor $U_{s}^{\prime}$ y $U_{s}$ se verifica $N=0,45 \times U_{c}$. Aplicando el mismo proceso de casos anteriores, esta ecuación equivale a

$$
Z_{4}=\frac{0,45^{2}}{0,35+r \times(1-\varrho)}
$$

\section{SECCIONES DE CANTO DADO}

Además de los parámetros previos, $f_{c}$, $\varrho$, son datos, en todos los problemas de secciones de canto dado, la cuaterna $(N, e, b, d)$ y el parámetro $r$. También, por consiguiente, es dato $U_{c}$. Se llama "punto selector» al determinado por las coordenadas

$$
P=\frac{N \times e}{d \times U_{c}}-r \times(1-\varrho) \quad ; \quad Q=\frac{N}{U_{c}}-r
$$

Los datos han de cumplir las condiciones de compatibilidad

$$
r \times(1-\varrho) \times d \times U_{c} \leqslant N \times e \leqslant \mu
$$

siendo

$$
\mu=\mu_{1}=\left[0,35 \times\left(1-\varrho^{2}\right)+r \times(1-\varrho)\right] \times d \times U_{c}
$$

en los casos de compresión, y

$$
\mu=\mu_{2}=[0,35+r \times(1-\varrho)] \times d \times U_{c}
$$

en los casos de flexión. Estas condiciones también son

$$
\begin{array}{ll}
0 \leqslant P \leqslant 0,35 \times\left(1-\varrho^{2}\right) & \text { para compresión, } \\
0 \leqslant P \leqslant 0,35 & \text { para flexión. }
\end{array}
$$

y así establecen los límites de la abscisa $P$ del punto selector.

3. Compresión, con la característica $P \leqslant 0,35 \times\left(1-\varrho^{2}\right)$.

3.1. Compresión compuesta. Es $q=1+\varrho$. Son incógnitas $c$ y $U_{s}$. En este caso las fórmulas [1.m], [1.r] adquieren las formas siguientes:

$$
\begin{aligned}
N \times e & =\left[0,50 \times c \times\left(1-\varrho^{2}\right)+r \times(1-\varrho)\right] \times d \times U_{c} \\
N & =[c \times(1+\varrho)+r] \times U_{c}+U_{s}
\end{aligned}
$$

equivalentes a las fórmulas

$$
c=\frac{2 \times P}{1-\varrho^{2}} \quad ; \quad U_{s}=\left(Q-\frac{2 \times P}{1-\varrho}\right) \times U_{c}
$$

con validez si es $U_{s} \geqslant 0$. La situación límite es $U_{s}=0$, equivale a la condición $Q \times$ $\times(1-\varrho)=2 \times P$, que es la ecuación de una línea recta en el campo de variabilidad del punto selector. 
3.2. Compresión interpolada. Es $U_{s}=0$. Como es $U_{s}^{\prime}=r \times U_{c}$, las incógnitas en este caso son $c$ y $q$.

La ecuación $[1 . \mathrm{m}]$ equivale a

$$
P=c \times q \times(1-0,50 \times q)
$$

La ecuación [1.r], con $U_{s}=0$, equivale a

$$
Q=c \times q
$$

Finalmente se obtiene

$$
q=2 \times \frac{Q-P}{Q} \quad ; \quad c=\frac{Q^{2}}{2 \times(Q-P)}
$$

Cuando el punto $(P, Q)$ está en la línea recta límite de la situación de compresión compuesta, la primera de estas dos fórmulas da $q=1+\varrho$ y la segunda $c=(2 \times \mathrm{P}) /\left(1-\varrho^{2}\right)$; es decir dicha línea recta es común a los dos campos de puntos selectores y es su frontera.

En cambio, si es $q=1$, de la primera fórmula se deduce $2 \times P=Q$, que es la ecuación de otra línea recta. Los puntos selectores situados entre esta recta $2 \times P=Q$ y la recta $2 \times P=Q \times(1-\varrho)$ corresponden a las secciones interpoladas en las que

$$
1 \leqslant q \leqslant 1+\varrho .
$$

4. Flexión, con la característica $P \leqslant 0,35$.

4.1. Flexión interpolada. Es $U_{s}=0$. También es $U_{s}^{\prime}=r \times U_{c}$; por consiguiente, como en el caso anterior de compresión interpolada, las incógnitas son $c$ y $q$. Fácilmente se comprueba que las ecuaciones son las mismas del caso anterior y también los resultados,

$$
q=2 \times \frac{Q-P}{Q} \quad, \quad c=\frac{Q^{2}}{2 \times(Q-P)}
$$

Una condición de borde de este campo es $q=1$, que conduce a la misma línea recta del anterior caso de compresión interpolada. Otra condición de borde es $c=1$, que, por la segunda fórmula, se convierte en la ecuación $P=Q \times(1-0,50 \times Q)$.

4.2. Flexión compuesta. Es $c=1$. Son incógnitas $q$ y $U_{s}$. Las fórmulas [1.m], [1.r] adquieren en este caso las formas siguientes:

$$
\begin{aligned}
N \times e & =[q \times(1-0,50 \times q)+r \times(1-\varrho)] \times d \times U_{c} \\
N & =(q+r) \times U_{c}-U_{s}
\end{aligned}
$$

equivalentes a las fórmulas

$$
P=q \times(1-0,50 \times q) \quad, \quad U_{s}=(q-Q) \times U_{c}
$$

con validez si es $U_{s} \geqslant 0$. La situación límite de esta condición es $U_{s}=0$, equivalente a $q=Q$, o sea, a la ecuación $P=Q \times(1-0,50 \times Q)$, que es idéntica a la obtenida en 4.1 como borde de los puntos selectores de flexión interpolada.

En resumen (véase la figura 3 , de $\varrho=0,10$ ), el campo de variabilidad del punto selector $(P, Q)$ queda dividido en cuatro recintos por las líneas siguientes:

(a) la recta de ecuación, $2 \times P=(1-\varrho) \times Q$

(b) la recta de ecuación, $2 \times P=Q$

(c) la curva de ecuación, $P=Q \times(1-0,50 \times Q)$.

- Por encima de la línea recta (a) todos los puntos selectores corresponden a compresión compuesta.

-- Entre las líneas rectas (a) y (b) todos los puntos selectores corresponden a compresión interpolada. 
- Entre la línea recta $(b)$ y la línea curva $(c)$ todos los puntos selectores corresponden a flexión interpolada.

- Entre la línea curva (c) y el eje coordenado de las $P$ todos los puntos selectores corresponden a flexión compuesta.

Finalmente, los puntos selectores que están en el contorno del campo de compresión en la recta $P=0,35 \times\left(1-\varrho^{2}\right)$ corresponden a secciones tope. Asimismo, corresponden a secciones tope los puntos situados en la recta $P=0,35$ del borde del campo de flexión.

\section{VALORES DEL PARAMETRO $r$ COMPATIBLES CON LA CUATERNA $(N, e, b, d)$}

Si los valores numéricos de $\varrho$ y de $f_{c}$ se han determinado previamente, a cada cuaterna de datos $N, e, b, d$, corresponden los infinitos puntos selectores dados por las coordenadas

$$
P=\frac{N \times e}{d \times U_{c}}-r \times(1-\varrho) \quad, \quad Q=\frac{N}{U_{c}}-r
$$

en las que $r$ puede asumir uno cualquiera de los valores del intervalo $(0,0,50)$.

Dichos puntos son los de un segmento rectilíneo cuyos extremos son:

$$
\begin{array}{lll}
P_{0}=\frac{N \times e}{d \times U_{c}} & , & Q_{0}=\frac{N}{U_{c}} \\
P_{1}=\frac{N \times e}{d \times U_{c}}-0,50 \times(1-\varrho) & , & Q_{1}=\frac{N}{U_{c}}-0,50
\end{array}
$$

Todos los segmentos así determinados son entre sí paralelos, y tienen igual longitud:

$$
L=0,50 \times \sqrt{1+(1-\varrho)^{2}}
$$

Los valores de $r$ compatibles con la cuaterna son los que corresponden a puntos selectores ubicados en el campo de variabilidad considerado en el párrafo II. Esta condición establece generalmente limitaciones al propio campo de variabilidad de $r$, que ya no será $(0,0,50)$, sino el que resulte de eliminar los valores de $r$ que dan puntos en el exterior (en el caso $\varrho=0,10$, siempre se tiene que disminuir dicho intervalo, porque no cabe completo, con su inclinación, entre los límites del campo). Es importante la obtención fácil de los valores compatibles de $r$, porque generalmente con $r$ se busca una armadura que satisfaga las ecuaciones del equilibrio en la rotura entre la carga y la sección dadas. Si puede obtenerse un valor menor de $r$ que también cumpla dicho equilibrio, éste será preferible al primero, y supone un aprovechamiento mayor de la sección dada de hormigón.

Puede sugerirse la construcción, para cada valor de $\varrho$, de una escalilla, de longitud

$$
L=0,50 \times \sqrt{1+(1-\varrho)^{2}}
$$

graduada convenientemente entre 0,50 y 0, y con una señal para su colocación angular.

\section{SECCIONES TOPE CON EL DATO $\varepsilon$}

El parámetro $\varepsilon$ es la distancia del punto de aplicación de $N$ al centro de la sección de hormigón. En general la carga exterior en una sección está constituida por un momento $M$ y una carga axil $N$. En tal caso es $\varepsilon=M: N$. Si se adopta como armadura $A_{s}^{\prime}$, la que resultaría comprimida al actuar solamente $M$, el parámetro $\varepsilon$ es siempre positivo. Existe la relación

$$
e=\varepsilon+0,50 \times(1-\varrho) \times d
$$

El canto útil de una sección tope se puede hallar en todos los casos con la fórmula

$$
N \times e=d^{2} \times b \times f_{c} \times[]
$$


como se ha visto repetidamente. Cuando se dispone del parámetro $\varepsilon$, y no del parámetro $e$, se puede escribir:

$$
N \times[\varepsilon+0,50 \times(1-\varrho) \times d]=d^{2} \times b \times f_{c} \times[]
$$

y, para determinar el canto útil $d$, hay que resolver esta ecuación algebraica de segundo grado, cuya raíz positiva es:

$$
d=\frac{0,25 \times(1-\varrho) \times N}{b \times f_{c} \times[]}+\sqrt{\left[\frac{0,25 \times(1-\varrho) \times N}{b \times f_{c} \times[]}\right]^{2}+\frac{N \times \varepsilon}{b \times f_{c} \times[]}}
$$

En el símbolo [ ] se ha de colocar el valor de uno de los dos polinomios

$$
0,35 \times\left(1-\varrho^{2}\right)+r \times(1-\varrho) \quad ; \quad 0,35+r \times(1-\varrho)
$$

según se compruebe que es un caso de compresión o de flexión.

Las dimensiones $b, d$, de las secciones de una estructura hiperestática están ligadas por relaciones que determinan dichas dimensiones $b, d$, una vez establecidas las de una de ellas. Por este motivo son más numerosos los casos de cálculo con el canto dado, y también, por consiguiente, los casos en que se dispone directamente del parámetro $e$.

\section{CLASIFICACION SINOPTICA DE LAS SECCIONES RECTANGULARES DE HORMIGON ARMADO}

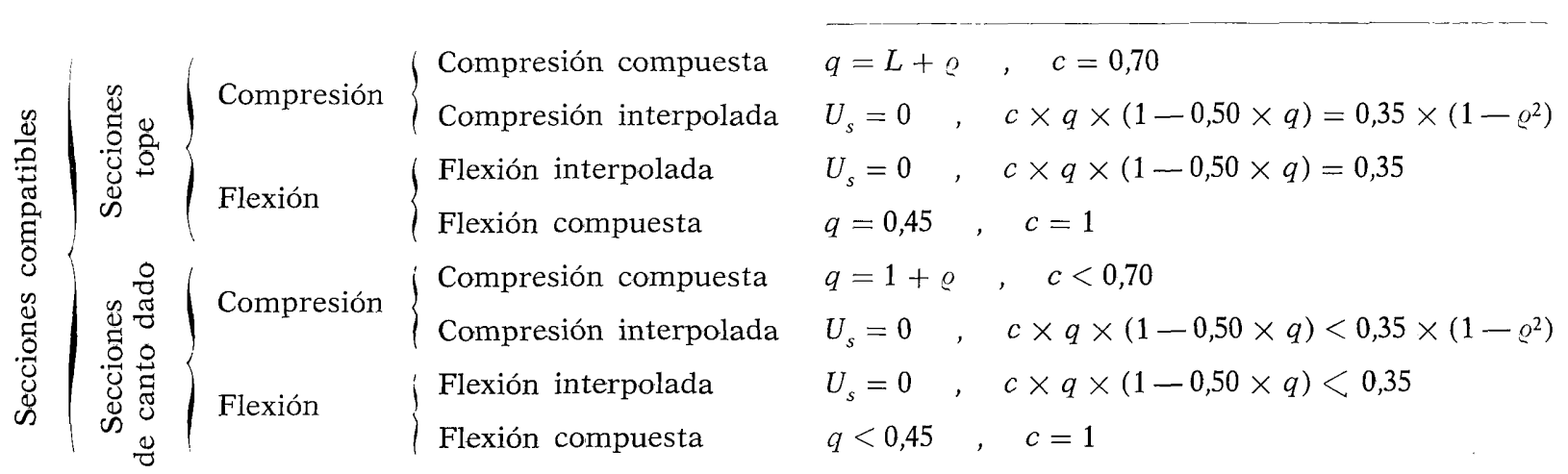

Las secciones tope, por un lado, y las secciones de canto dado, por otro distinto, son susceptibles, una vez determinados numéricamente los parámetros previos $\varrho$ y $f_{c}$, de una representación gráfica, en virtud de la cual, a cada una de sus cuatro modalidades corresponde un recinto plano característico. Las dos coordenadas del "punto selector», obtenidas sencilla e inmediatamente a partir de los datos de cada caso, ubican a dicho punto selector en el recinto que corresponde a su proceso de cálculo.

\section{E.JEMPLOS NUMERICOS}

Se emplean los siguientes coeficientes numéricos:

- Mayoración de la carga:

$$
\begin{aligned}
& \gamma_{N}=+1,60 \\
& \gamma_{C}=-1,50 \\
& \gamma_{S}=-1,15
\end{aligned}
$$

- Minoración para el acero:

- Coeficiente de rotura del hormigón: $150 \mathrm{kp} / \mathrm{cm}^{2}$

- Coeficiente de fluencia del acero: $4.000 \mathrm{kp} / \mathrm{cm}^{2}$

Los coeficientes de los materiales cumplen el artículo 28.3 de la Instrucción. Por consiguiente, se adoptan

$$
\begin{aligned}
& f_{c}=150: 1,50=100 \mathrm{kp} / \mathrm{cm}^{2} \\
& f_{s}=4.000: 1,15=3.478 \mathrm{kp} / \mathrm{cm}^{2}
\end{aligned}
$$


Secciones rectangulares tope (véase fig. 2).

\section{Ejemplo 1. ${ }^{\circ}$}

Datos:

$$
\begin{aligned}
N & =1,60 \times 90.000=144.000 \mathrm{kp} \quad, \quad e=22 \mathrm{~cm} \\
b & =30 \mathrm{~cm} \quad, \quad \varrho=0,10 \\
Z & =\frac{144.000}{30 \times 22 \times 100}=2,18
\end{aligned}
$$

Incógnitas:

$$
d \quad, \quad U_{s}
$$

Según la figura 2, para todos los valores de $r$ a este valor $Z$ corresponde compresión compuesta. Si $r$ está en el intervalo $(0,0,105)$ será $U_{s}^{\prime}<U_{s}$. En el intervalo $(0,105,0,50)$ de $r$ es $U_{s}^{\prime}>U_{s}$.

CASo (a). Se ha elegido $r=0,10$. Resultados:

$$
\begin{aligned}
d & =\sqrt{\frac{144.000 \times 22}{30 \times 100 \times(0,35 \times 0,99+0,10 \times 0,90)}}=\sqrt{\frac{3.168 .000}{3.000 \times 0,4365}}=49,19 \mathrm{~cm} \\
U_{c} & =147.557 \mathrm{kp} \quad, \quad U_{s}^{\prime}=0,10 \times 147.557=14.756 \mathrm{kp} \\
U_{s} & =144.000-(0,77+0,10) \times 147.557=15.625 \mathrm{kp}
\end{aligned}
$$

Comprobación de las resultantes:

$$
30 \times 49,19 \times 1,1 \times 70+14.756+15.625=113.629+30.381=144.010 \mathrm{kp}
$$

La diferencia en $\%$ es:

$$
(144.010-144.000) \times 100: 144.010=0,007 \%
$$

Comprobación de los momentos (con $d^{\prime}=49,19 \times 0,10=4,92 \mathrm{~cm}$ ):

$$
\begin{gathered}
30 \times 70 \times 0,50 \times 49,19^{2}-30 \times 70 \times 0,50 \times 4,92^{2}+14.756 \times 0,9 \times 49,19= \\
=2.540 .639-25.417+653.263=3.168 .485 \mathrm{~cm} \cdot \mathrm{kp}
\end{gathered}
$$

La diferencia en \% es:

$$
(3.168 .485-3.168 .000) \times 100: 3.168 .485=0,015 \%
$$

Caso (b). Se elige $r=0,105$ (éste es un valor no calculado, sino «leído» en el gráfico de la figura 2). Resultados:

$$
\begin{aligned}
d & =\sqrt{\frac{144.000 \times 22}{30 \times 100 \times[0,35 \times 0,99+0,105 \times 0,90]}}=\sqrt{\frac{3.168 .000}{3.000 \times 0,441}}=48,93 \mathrm{~cm} \\
U_{c} & =146.803 \mathrm{kp} \quad, \quad U_{s}^{\prime}=0,105 \times 146.803=15.414 \mathrm{kp} \\
U_{s} & =144.000-(0,77+0,105) \times 146.803=15.547 \mathrm{kp}
\end{aligned}
$$

La diferencia en \% de ambas armaduras es:

$$
(15.547-15.414) \times 100: 15.547=0,86 \%
$$

Comprobación de las resultantes:

$$
30 \times 48,93 \times 1,1 \times 70+15.414+15.547=113.028+30.961=143.989 \mathrm{kp}
$$

La diferencia en \% es:

$$
(144.000-143.989) \times 100: 144.000=0,008 \%
$$


Comprobación de los momentos:

$$
\begin{gathered}
30 \times 70 \times 0,50 \times\left(48,93^{2}-4,893^{2}\right)+15.414 \times 0,90 \times 48,93= \\
=2.488 .714+678.786=3.167 .500 \mathrm{~cm} \cdot \mathrm{kp}
\end{gathered}
$$

La diferencia en \% es:

$$
(3.168 .000-3.167 .500) \times 100: 3.168 .000=0,016 \%
$$

Caso (c). Se elige $r=0,35$. Resultados:

$$
\begin{aligned}
d & =\sqrt{\frac{144.000 \times 22}{30 \times 100 \times(0,35 \times 0,99+0,35 \times 0,90)}}=\sqrt{\frac{3.168 .000}{3.000 \times 0,6615}}=39,95 \mathrm{~cm} \\
U_{c} & =119.864 \mathrm{kp} \quad, \quad U_{s}^{\prime}=0,35 \times 119.864=41.952 \mathrm{kp} \\
U_{s} & =144.000-(0,77+0,35) \times 119.864=9.752 \mathrm{kp}
\end{aligned}
$$

Comprobación de las resultantes:

$$
30 \times 39,95 \times 1,1 \times 70+41.952+9.752=143.989 \mathrm{kp}
$$

La diferencia en \% es:

$$
(144.000-143.989) \times 100: 144.000=0,008 \%
$$

Comprobación de los momentos:

$$
\begin{gathered}
30 \times 70 \times 0,50 \times 39,95^{2} \times 0,99+41.952 \times 0,90 \times 39,95= \\
=1.659 .045+1.508 .384=3.167 .429 \mathrm{~cm} \cdot \mathrm{kp}
\end{gathered}
$$

La diferencia en \% es:

$$
(3.168 .000-3.167 .429) \times 100: 3.168 .000=0,02 \%
$$

\section{Ejemplo 2.}

Datos:

$$
\begin{aligned}
& \mathrm{N}=1,60 \times 90.000=144.000 \mathrm{kp} \quad, \quad e=26 \mathrm{~cm} \\
& b=30 \mathrm{~cm}, \quad \varrho=0,10 \\
& Z=\frac{144.000}{30 \times 26 \times 100}=1,846
\end{aligned}
$$

Según el gráfico clasificador de la figura 2 al valor $Z=1,846$ del parámetro selector corresponden el intervalo $(0,0,285)$ de $r$ con situaciones de compresión compuesta y el intervalo $(0,285,0,50)$ de $r$ con situaciones de compresión interpolada.

Caso (a). Se elige $r=0,20$. Las incógnitas son $d$ y $U_{s}$. Resultados:

$$
\begin{aligned}
d & =\sqrt{\frac{144.000 \times 26}{30 \times 100 \times(0,35 \times 0,99+0,20 \times 0,90)}}=\sqrt{\frac{3.744 .000}{3.000 \times 0,5265}}=48,69 \mathrm{~cm} \\
U_{c} & =146.059 \mathrm{kp} \quad, \quad U_{s}^{\prime}=0,20 \times 146.059=29.212 \mathrm{kp} \\
U_{s} & =144.000-(0,77+0,20) \times 146.059=2.323 \mathrm{kp}
\end{aligned}
$$

Comprobación de las resultantes:

$$
30 \times 48,69 \times 1,1 \times 70+29.212+2.323=144.009 \mathrm{kp}
$$

La diferencia en \% es:

$$
(144.009-144.000) \times 100: 144.009=0,006 \%
$$


Comprobación de los momentos:

$$
\begin{gathered}
30 \times 70 \times 0,50 \times\left(48,69^{2}-4,869^{2}\right)+29.212 \times 0,90 \times 48,69= \\
=2.464 .359+1.280 .099=3.744 .458 \mathrm{~cm} \cdot \mathrm{kp}
\end{gathered}
$$

La diferencia en $\%$ es:

$$
(3.744 .458-3.744 .000) \times 100: 3.744 .458=0,012 \%
$$

CAso (b). Se elige $r=0,285$. Las incógnitas son $d$ y $U_{s}$. Resultados:

$$
\begin{aligned}
& d=\sqrt{\frac{144.000 \times 26}{30 \times 100 \times(0,35 \times 0,99+0,285 \times 0,90)}}=\sqrt{\frac{3.744 .000}{3.000 \times 0,603}}=45,49 \mathrm{~cm} \\
& U_{c}=136.480 \mathrm{kp} \quad ; \quad U_{s}^{\prime}=0,285 \times 136.480=38.897 \mathrm{kp} \\
& U_{s}=144.000-(0,77+0,285) \times 136.480=14 \mathrm{kp} \simeq 0
\end{aligned}
$$

Comprobación de las resultantes:

$$
30 \times 45,49 \times 1,1 \times 70+38.897+14=143.993 \mathrm{kp} .
$$

La diferencia en \% es:

$$
(144.000-143.993) \times 100: 144.000=0,005 \% .
$$

Comprobación de los momentos:

$$
\begin{aligned}
30 \times 70 \times 0,50 \times 45,49^{2} \times 0,99 & +38.897 \times 0,90 \times 45,49=2.151 .079+1.592 .482= \\
& =3.743 .561 \mathrm{~cm} \cdot \mathrm{kp} .
\end{aligned}
$$

La diferencia en $\%$ es:

$$
(3.744 .000-3.743 .561) \times 100: 3.744 .000=0,012 \% .
$$

Caso (c). Se elige $r=0,40$. Las incógnitas son $d, q, c$.

Resultados:

$$
\begin{gathered}
d=\sqrt{\frac{144.000 \times 26}{30 \times 100 \times(0,35 \times 0,99+0,40 \times 0,90)}}=\sqrt{\frac{3.744 .000}{3.000 \times 0,7065}}=42,03 \mathrm{~cm} \\
U_{c}=126.088 \mathrm{kp} ; U_{s}=0 ; U_{s}^{\prime}=0,40 \times 126.088=50.435 \mathrm{kp} \\
c \times q=\frac{144.000}{126.088}-0,40=0,742 \\
1-0,50 \times q=0,35 \times 0,99: 0,742=0,467 \\
q=2 \times(1-0,467)=1,066<1+\varrho \\
c=0,742: 1,066=0,696
\end{gathered}
$$

Comprobación de las resultantes:

$$
30 \times 1,066 \times 42,03 \times 69,60+50.435=143.986 \mathrm{kp}
$$

La diferencia en \% es:

$$
(144.000-143.986) \times 100: 144.000=0,01 \%
$$

Comprobación de los momentos:

$$
(\delta=0,066 \times 42,03=2,774 \mathrm{~cm})
$$

$$
\begin{gathered}
30 \times 69,60 \times 0,50 \times\left(42,03^{2}-2,774^{2}\right)+50.435 \times 0,90 \times 42,03=1.836 .214+1.907 .805= \\
3.744 .019 \mathrm{~cm} \cdot \mathrm{kp}
\end{gathered}
$$

La diferencia en $\%$ es:

$$
(3.744 .019-3.744 .000) \times 100: 3.744 .019=0,001 \%
$$




\section{Ejemplo 3.}

Datos:

$$
\begin{aligned}
N & =1,60 \times 90.000=144.000 \mathrm{kp} \quad ; \quad e=26 \mathrm{~cm} \\
b & =35 \mathrm{~cm} \quad ; \quad \varrho=0,10 \\
Z & =\frac{144.000}{35 \times 26 \times 100}=1,58
\end{aligned}
$$

En el gráfico de la figura 2 se ve que en el intervalo $(0,0,28)$ de $r$ las correspondientes secciones están en situación de compresión interpolada; mientras que en el intervalo $(0,263$, $0,50)$ la situación es de flexión interpolada. Los puntos selectores del intervalo $(0,263,0,280)$ corresponden indistintamente a una de estas dos situaciones, y se puede preferir la primera porque da valores ligeramente mayores.

Caso (a). Se elige $r=0,15$. Las incógnitas son $d, q, c$.

Compresión interpolada. Resultados:

$$
\begin{aligned}
& d=\sqrt{\frac{144.000 \times 26}{35 \times 100 \times(0,35 \times 0,99+0,15 \times 0,90)}}=\sqrt{\frac{3.744 .000}{3.500 \times 0,4815}}=47,13 \mathrm{~cm} \\
& U_{c}=164.970 \mathrm{kp} ; U_{s}=0 ; U_{s}^{\prime}=0,15 \times 164.970=24.745 \mathrm{kp} \\
& c \times q=\frac{144.000}{164.970}-0,15=0,723 \\
& 1-0,50 \times q=0,35 \times 0,99: 0,723=0,479 \\
& q=2 \times(1-0,479)=1,042<1+\varrho \\
& c=0,723: 1,042=0,6939
\end{aligned}
$$

Comprobación de las resultantes:

$$
35 \times 1,042 \times 47,13 \times 69,39+24.745=144.015 \mathrm{kp}
$$

La diferencia en \% es:

$$
(144.015-144.000) \times 100: 144.015=0,01 \%
$$

Comprobación de los momentos ( $\operatorname{con} \delta=0,042 \times 47,13=1,98 \mathrm{~cm}$ )

$$
\begin{gathered}
35 \times 69,39 \times 0,50 \times\left(47,13^{2}-1,98^{2}\right)+24.745 \times 0,90 \times 47,13= \\
=2.692 .543+1.049 .609=3.742 .152 \mathrm{~cm} \cdot \mathrm{kp}
\end{gathered}
$$

La diferencia en \% es:

$$
(3.744 .000-3.742 .152) \times 100: 3.744 .000=0,05 \%
$$

Caso $\left(b_{1}\right)$. Se elige $r=0,27$. Proceso en compresión interpolada. Incógnitas: $d, q, c$.

Resultados:

$$
\begin{gathered}
d=\sqrt{\frac{144.000 \times 26}{35 \times 100 \times(0,35 \times 0,99+0,27 \times 0,90)}}=\sqrt{\frac{3.744 .000}{3.500 \times 0,5895}}=42,60 \mathrm{~cm} \\
U_{c}=149.094 \mathrm{kp} ; U_{s}=0 ; U_{s}^{\prime}=0,27 \times 149.094=40.255 \mathrm{kp} \\
c \times q=\frac{144.000-0,27=0,696}{149.094-0,27 \times} \quad \begin{array}{c}
1-0,50 \times q=0,35 \times 0,99: 0,696=0,498 \\
q=2 \times(1-0,498)=1,004 ; c=0,696: 1,004=0,6932 .
\end{array}
\end{gathered}
$$

Caso $\left(b_{2}\right)$. Se elige $r=0,27$. Proceso de flexión interpolada. Incógnitas: $d, q, c$. Resultados:

$$
\begin{aligned}
d= & \sqrt{\frac{144.000 \times 26}{35 \times 100 \times(0,35+0,27 \times 0,9)}}=\sqrt{\frac{3.744 .000}{3.500 \times 0,593}}=42,47 \mathrm{~cm} \\
U_{c}= & 148.653 \mathrm{kp} ; U_{s}=0 ; U_{s}^{\prime}=0,27 \times 148.653=40.136 \mathrm{kp} \\
c \times q= & \frac{144.000}{148.653}-0,27=0,699 \\
& q=2 \times(1-0,501)=0,998<1 ; c=0,699: 0,998=0,7004 .
\end{aligned}
$$


Caso (c). Se elige $r=0,40$. Flexión interpolada. Incógnitas: $d, q, c$. Resultados:

$$
\begin{gathered}
d=\sqrt{\frac{144.000 \times 26}{35 \times 100 \times(0,35+0,40 \times 0,90)}}=\sqrt{\frac{3.744 .000}{3.500 \times 0,71}}=38,82 \mathrm{~cm} \\
U_{c}=135.854 \mathrm{kp} ; U_{s}=0 ; U_{s}^{\prime}=0,40 \times 135.854=54.342 \mathrm{kp} \\
c \times q=\frac{144.000}{135.854}-0,40=0,660 \\
\quad 1-0,50 \times q=0,35: 0,660=0,530 \\
q=2 \times(1-0,530)=0,94 ; c=0,660: 0,940=0,7021 .
\end{gathered}
$$

Comprobación de las resultantes:

$$
35 \times 0,94 \times 38,82 \times 70,21+54.342=144.013 \mathrm{kp}
$$

La diferencia en \% es:

$$
(144.013-144.000) \times 100: 144.013=0,01 \%
$$

Comprobación de los momentos:

$$
\begin{gathered}
35 \times 0,94 \times 38,82^{2} \times 70,21 \times(1-0,50 \times 0,94)+54.342 \times 0,90 \times 38,82= \\
=1.844 .938+1.898 .601=3.743 .539 \mathrm{~cm} \cdot \mathrm{kp}
\end{gathered}
$$

La diferencia en $\%$ es:

$$
(3.744 .000-3.743 .539) \times 100: 3.744 .000=0,012 \%
$$

\section{Ejemplo $4 .^{\circ}$}

Datos:

$$
\begin{aligned}
N & =1,60 \times 60.000=96.000 \mathrm{kp} ; e=33 \mathrm{~cm} \\
b & =35 \mathrm{~cm} ; \varrho=0,10 \\
Z & =\frac{96.000}{35 \times 33 \times 100}=0,83
\end{aligned}
$$

A este valor del parámetro selector corresponden en la figura 2 un intervalo $(0,0,225)$ de valores de $r$ con secciones en flexión interpolada y otro $(0,225,0,50)$ con secciones en flexión compuesta.

Caso (a). Se elige $r=0,15$. Flexión interpolada. Incógnitas: $d, q, c$. Resultados:

$$
\begin{gathered}
d=\sqrt{\frac{96.000 \times 33}{35 \times 100 \times(0,35+0,15 \times 0,90)}}=\sqrt{\frac{3.168 .000}{3.500 \times 0,485}}=43,20 \mathrm{~cm} \\
U_{c}=141.201 \mathrm{kp} ; U_{s}=0 ; U_{s}^{\prime}=0,15 \times 151.201=22.680 \mathrm{kp} \\
c \times q=\frac{96.000}{151.201}-0,15=0,485 \\
\quad 1-0,50 \times q=0,35: 0,485=0,722 \\
q=2 \times(1-0,722)=0,556 ; \quad c=0,485: 0,556=0,8723 .
\end{gathered}
$$

Comprobación de las resultantes:

$$
35 \times 0,556 \times 43,20 \times 87,23+22.680=96.012 \mathrm{kp}
$$

La diferencia en \% es:

$$
(96.012-96.000) \times 100: 96.012=0,012 \%
$$

Comprobación de los momentos:

$$
\begin{gathered}
35 \times 0,556 \times 43,20^{2} \times 87,23 \times(1-0,50 \times 0,556)+22.680 \times 0,90 \times 43,20= \\
=2.287 .249+881.798=3.169 .047 \mathrm{~cm} \cdot \mathrm{kp}
\end{gathered}
$$


La diferencia en \% es:

$$
(3.169 .047-3.168 .000) \times 100: 3.169 .047=0,033 \%
$$

Caso (b). Se elige $r=0,375$. Flexión compuesta. Incógnitas: $d, U_{s}$.

$$
\begin{aligned}
d & =\sqrt{\frac{96.000 \times 33}{35 \times 100 \times(0,35+0,375 \times 0,90)}}=\sqrt{\frac{3.168 .000}{3.500 \times 0,6875}}=36,28 \mathrm{~cm} \\
U_{c} & =126.996 \mathrm{kp} ; U_{s}^{\prime}=0,375 \times 126.996=47.624 \mathrm{kp} \\
U_{s} & =(0,45+0,375) \times 126.996-96.000=8.772 \mathrm{kp} .
\end{aligned}
$$

Comprobación de las resultantes:

$$
35 \times 0,45 \times 36,28 \times 100+47.624-8.772=95.994 \mathrm{kp}
$$

La diferencia en $\%$ es:

$$
(96.000-95.994) \times 100: 96.000=0,006 \%
$$

Comprobación de los momentos:

$$
\begin{gathered}
35 \times 0,45 \times 36,28^{2} \times 100 \times(1-0,50 \times 0,45)+47.624 \times 0,90 \times 36,28= \\
=1.606 .633+1.555 .019=3.161 .652 \mathrm{~cm} \cdot \mathrm{kp}
\end{gathered}
$$

La diferencia en \% es:

$$
(3.168 .000-3.161 .652) \times 100: 3.168 .000=0,2 \%
$$

\section{Ejemplo 5.}

Datos:

$$
\begin{aligned}
N & =1,60 \times 60.000=96.000 \mathrm{kp} ; \quad e=72 \mathrm{~cm} \\
b & =35 \mathrm{~cm} ; \quad \varrho=0,10 \\
Z & =\frac{96.000}{35 \times 72 \times 100}=0,38 .
\end{aligned}
$$

A este valor del parámetro selector corresponden todos los valores de $r$ entre 0 y 0,50 con secciones en situación de flexión compuesta. En particular, al valor $r=0,20$ le corresponden armaduras simétricas.

Se elige $r=0,20$. Las incógnitas son $d$ y $U_{s}=U^{\prime}{ }_{s}$.

$$
\begin{aligned}
d & =\sqrt{\frac{96.000 \times 72}{35 \times 100 \times(0,35+0,20 \times 0,90)}}=\sqrt{\frac{6.912 .000}{3.500+0,53}}=61,04 \mathrm{~cm} \\
U_{c} & =213.648 \mathrm{kp} ; U_{s}^{\prime}=0,2 \times 213.648=42.730 \mathrm{kp} \\
U_{s} & =(0,45+0,20) \times 213.648-96.000=42.871 \mathrm{kp} .
\end{aligned}
$$

La diferencia de ambas armaduras en \% es:

$$
(42.871-42.730) \times 100: 42.871=0,33 \%
$$

Comprobación de las resultantes:

$$
35 \times 0,45 \times 61,04 \times 100+42.871-42.730=96.279 \mathrm{kp} .
$$

La diferencia en \% es:

$$
(96.279-96.000) \times 100: 96.279=0,29 \%
$$

Comprobación de los momentos:

$$
\begin{gathered}
35 \times 0,45 \times 61,04^{2} \times 100 \times(1-0,5 \times 0,45)+42.730 \times 0,90 \times 61,04= \\
=4.547 .904+2.347 .415=6.895 .319 \mathrm{~cm} \cdot \mathrm{kp}
\end{gathered}
$$


La diferencia en \% es:

$$
(6.912 .000-6.895 .319) \times 100: 6.912 .000=0,24 \%
$$

Secciones de canto dado (véase fig. 3).

\section{Ejemplo $60^{\circ}$}

Datos:

$$
\begin{aligned}
N & =1,60 \times 100.000=160.000 \mathrm{kp} \\
e & =25 \mathrm{~cm} \\
r & =0,30 \\
b & =30 \mathrm{~cm} \\
d & =50 \mathrm{~cm} \\
\varrho & =0,10
\end{aligned}
$$

Punto selector:

$$
\begin{aligned}
U_{c} & =30 \times 50 \times 100=150.000 \mathrm{kp} . \\
P & =\frac{160.000 \times 25}{50 \times 150.000}-0,30 \times 0,90=0,263<0,35 \times 0,99=0,3465 \\
Q & =\frac{160.000}{150.000}-0,30=0,767 .
\end{aligned}
$$

En el gráfico de la figura 3 el punto $P, Q$ está situado en el campo de compresión compuesta. Incógnitas: $c$ y $U_{s}$.

Resultados:

$$
\begin{aligned}
c & =\frac{2 \times 0,263}{0,99}=0,5313 ; U^{\prime}=0,30 \times 150.000=45.000 \mathrm{kp} \\
U_{s} & =\left(0,767-\frac{2 \times 0,263}{0,90}\right) \times 150.000=27.383 \mathrm{kp} .
\end{aligned}
$$

Comprobación de las resultantes:

$$
30 \times 1,1 \times 50 \times 53,13+45.000+27.383=160.048 \mathrm{kp} .
$$

La diferencia en $\%$ es:

$$
(160.048-160.000) \times 100: 160.048=0,03 \%
$$

Comprobación de los momentos $(\delta=50 \times 0,10=5,00 \mathrm{~cm})$ :

$$
\begin{gathered}
30 \times 53,13 \times 50^{2} \times 0,50 \times\left(1-0,10^{2}\right)+45.000 \times 0,90 \times 50= \\
=1.972 .451+2.025 .000=3.997 .451 \mathrm{~cm} \cdot \mathrm{kp} \\
N \times e=160.000 \times 25=4.000 .000 \mathrm{~cm} \cdot \mathrm{kp}
\end{gathered}
$$

La diferencia en \% es:

$$
(4.000 .000-3.997 .451) \times 100: 4.000 .000=0,06 \% .
$$

\section{Ejemplo 7.}

Datos:

$$
\begin{aligned}
N & =1,60 \times 100.000=160.000 \mathrm{kp} \\
e & =34 \mathrm{~cm} \\
r & =0,50 \\
b & =30 \mathrm{~cm} \\
d & =50 \mathrm{~cm} \\
\varrho & =0,10 \\
U_{c} & =30 \times 50 \times 100=150.000 \mathrm{kp}
\end{aligned}
$$


Punto selector:

$$
\begin{aligned}
P & =\frac{160.000 \times 34}{50 \times 150.000}-0,50 \times 0,90=0,275 \\
Q & =\frac{160.000}{150.000}-0,50=0,567
\end{aligned}
$$

Según la figura 3 el punto selector está en la parte de compresión interpolada. Es $U_{s}=0$. Incógnitas, $q$ y $c$.

Resultados:

$$
\begin{gathered}
U^{\prime}{ }_{s}=0,50 \times 150.000=75.000 \mathrm{kp} \\
q=2 \times \frac{0,567-0,275}{0,567}=1,03 \quad ; \quad(1<1,03<1,1) \\
c=\frac{0,567^{2}}{2 \times(0,567-0,275)}=0,5505
\end{gathered}
$$

Comprobación de las resultantes:

$$
30 \times 1,03 \times 50 \times 55,05+75.000=160.052 \mathrm{kp}
$$

La diferencia en \% es:

$$
(160.052-160.000) \times 100: 160.052=0,03 \%
$$

Comprobación de los momentos $(\delta=0,03 \times 50=1,5 \mathrm{~cm})$ :

$$
\begin{gathered}
30 \times 55,05 \times 0,50 \times\left(50^{2}-1,5^{2}\right)+75.000 \times 0,90 \times 50= \\
=2.062 .517+3.375 .000=5.437 .517 \mathrm{~cm} \cdot \mathrm{kp} \\
N \times e=160.000 \times 34=5.440 .000 \mathrm{~cm} \cdot \mathrm{kp}
\end{gathered}
$$

La diferencia en \% es:

$$
(5.440 .000-5.437 .517) \times 100: 5.440 .000=0,05 \%
$$

\section{Ejemplo 8.}

Datos:

$$
\begin{aligned}
N & =1,6 \times 47.000=75.200 \mathrm{kp} \\
e & =35 \mathrm{~cm} \\
r & =0,15 \\
b & =30 \mathrm{~cm} \\
d & =50 \mathrm{~cm} \\
\varrho & =0,10 \\
U_{c} & =30 \times 50 \times 100=150.000 \mathrm{kp}
\end{aligned}
$$

Punto selector:

$$
\begin{aligned}
& P=\frac{75.200 \times 35}{50 \times 150.000}-0,15 \times 0,90=0,216 \\
& Q=\frac{75.200}{150.000}-0,15=0,351
\end{aligned}
$$

Según el gráfico clasificador de las secciones de canto dado de la figura 3, la sección correspondiente a estos datos está en flexión interpolada. Es $U_{s}=0$. Las incógnitas son $q$ y $c$. 
Resultados:

$$
\begin{gathered}
q=2 \times \frac{0,351-0,216}{0,351}=0,769 \quad ; \quad 0,45<0,769<1,00 \\
c=\frac{0,351^{2}}{2 \times(0,351-0,216)}=0,4563
\end{gathered}
$$

Con $U_{s}=0 \quad ; \quad U_{s}^{\prime}=0,15 \times 150.000=22.500 \mathrm{kp}$

Comprobación de las resultantes:

$$
30 \times 0,769 \times 50 \times 45,63+22.500=75.134 \mathrm{kp}
$$

La diferencia en \% es:

$$
(75.200-75.134) \times 100: 75.200=0,09 \%
$$

Comprobación de los momentos:

$$
\begin{aligned}
& 30 \times 0,769 \times 50^{2} \times 45,63 \times(1-0,50 \times 0,769)+22.500 \times 0,90 \times 50= \\
&= 1.619 .818+1.012 .500=2.632 .318 \mathrm{~cm} \cdot \mathrm{kp} \\
& N \times e=75.200 \times 35=2.632 .000 \mathrm{~cm} \cdot \mathrm{kp}
\end{aligned}
$$

La diferencia en \% es:

$$
(2.632 .318-2.632 .000) \times 100: 2.632 .318=0,012 \%
$$

\section{Ejemplo 9.}

Datos:

$$
\begin{aligned}
N & =1,6 \times 56.250=90.000 \mathrm{kp} \\
e & =68 \mathrm{~cm} \\
r & =0,35 \\
b & =30 \mathrm{~cm} \\
d & =60 \mathrm{~cm} \\
\varrho & =0,10 \\
U_{c} & =30 \times 60 \times 100=180.000 \mathrm{kp}
\end{aligned}
$$

Punto selector:

$$
\begin{aligned}
& P=\frac{90.000 \times 68}{60 \times 180.000}-0,35 \times 0,90=0,252 \\
& Q=\frac{90.000}{180.000}-0,35=0,15
\end{aligned}
$$

Según la situación de este punto en el gráfico de la figura 3, la sección es de flexión compues. ta. Sus incógnitas son $q$ y $U_{s}$.

Resultados:

$$
q \times(1-0,50 \times q)=0,252,
$$

o sea:

$$
\begin{gathered}
q=1 \pm \sqrt{0,496}=0,296 \\
U_{s}=(0,296+0,35) \times 180.000-90.000=26.280 \mathrm{kp} \\
U_{s}^{\prime}=0,35 \times 180.000=63.000 \mathrm{kp}
\end{gathered}
$$

Comprobación de las resultantes:

$$
30 \text { } 0,296 \times 60 \times 100 \dashv 63.000-26.280=90.000 \mathrm{kp}
$$


Comprobación de los momentos:

$$
\begin{gathered}
30 \times 0,296 \times 60^{2} \times 100 \times(1-0,50 \times 0,296)+63.000 \times 0,90 \times 60= \\
=2.723 .674+3.402 .000=6.125 .674 \mathrm{~cm} \cdot \mathrm{kp} \\
N \times e=90.000 \times 68=6.120 .000
\end{gathered}
$$

La diferencia en \% es:

$$
(6.125 .674-6.120 .000) \times 100: 6.125 .674=0,09 \%
$$

\section{Ejemplo 10. ${ }^{\circ}$}

Hallar los intervalos de compatibilidad del parámetro $r$ en los ejemplos anteriores:

a) Ejemplo 6.0:

$$
\begin{gathered}
P_{0}=\frac{160.000 \times 25}{50 \times 150.000}=0,533 \quad ; \quad Q_{0}=\frac{160.000}{150.000}=1,067 \\
P_{1}=0,533-0,50 \times 0,90=0,083 \\
Q_{1}=1,067-0,50=0,567
\end{gathered}
$$

El punto $\left(P_{0}, Q_{0}\right)$ es exterior, y el punto $\left(P_{1}, Q_{1}\right)$ es interior.

El valor de $r$ de la intersección verifica la ecuación $0,533-r \times 0,90=0,35 \times\left(1-\varrho^{2}\right)=0,35 \times$ $\times 0,99=0,3465$, o sea:

$$
r=(0,533-0,3465): 0,90=0,207
$$

y resulta:

$$
Q=1,067-0,207=0,86
$$

El intervalo de compatibilidad de $r$ es $(0,207,0,50)$.

Por consiguiente, la solución óptima es la sección tope en compresión compuesta:

$$
\begin{aligned}
b & =30 \mathrm{~cm} \\
d & =50 \mathrm{~cm} \\
r & =0,207 \\
U_{s}^{\prime} & =0,207 \times 150.000=31.050 \mathrm{kp} \\
U_{s} & =160.000-(0,77+0,207) \times 150.000=13.460 \mathrm{kp}
\end{aligned}
$$

La suma de estas dos armaduras es:

$$
31.050+13.460=44.510 \mathrm{kp}
$$

mientras que la suma de las dos armaduras del ejemplo $6 .^{\circ}$ es:

$$
45.000+27.383=72.383 \mathrm{kp}
$$

b) Ejemplo 7.:

$$
\begin{gathered}
P_{0}=\frac{160.000 \times 34}{50 \times 150.000}=0,725 \quad ; \quad Q_{0}=\frac{160.000}{150.000}=1,067 \\
P_{1}=0,725-0,45=0,275 \\
Q_{1}=1,067-0,50=0,567
\end{gathered}
$$

La intersección corresponde a

$$
r=(0,725-0,35): 0,90=0,417
$$

con

$$
Q=1,067-0,417=0,65
$$


Corresponde a este valor del parámetro $r$ una sección tope en situación de flexión interpolada, cuyos resultados son:

$$
\begin{aligned}
U_{s} & =0 \\
U_{s}^{\prime} & =0,417 \times 150.000=62.550 \mathrm{kp} \\
c \times q & =1,067-0,417=0,65 \\
1-0,50 \times q & =0,35: 0,65=0,538 \\
q & =2-1,076=0,924 \\
c & =0,65: 0,924=0,7034
\end{aligned}
$$

La suma de las armaduras en la presente solución es:

$$
U_{s}^{\prime}+U_{s}=62.550 \mathrm{kp}
$$

mientras que en la solución del ejemplo $7 .^{\circ}$ es:

$$
U^{\prime}{ }^{\prime}+U_{s}=75.000 \mathrm{kp}
$$

El intervalo de compatibilidad de $r$ es $(0,417,0,50)$.

c) Ejemplo 8. :

$$
P_{0}=\frac{75.200 \times 35}{50 \times 150.000}=0,35 \quad ; \quad Q_{0}=\frac{75.200}{150.000}=0,50
$$

El valor mínimo compatible de $r$ es 0 . El punto $\left(P_{0}, Q_{0}\right)$ se halla en el borde de flexión interpolada y en situación de momento tope.

Los resultados son:

$$
U_{s}=0 \quad ; \quad U_{s}^{\prime}=0 \quad ; \quad q=2 \times \frac{0,50-0,35}{0,50}=0,6 \quad ; \quad c=\frac{0,50}{0,60}=0,8333
$$

d) Ejemplo 9.:

$$
P_{0}=\frac{90.000 \times 68}{60 \times 180.000}=0,567 \quad ; \quad Q_{0}=\frac{90.000}{180.000}=0,50
$$

En este caso el valor mínimo compatible de $r$ viene dado por la ecuación

$$
0,567-r: 0,90=0,35
$$

o sea:

$$
r=0,241
$$

Con este valor de $r$ son:

$$
P=0,35 ; Q=0,259
$$

Corresponde a este punto selector una sección en flexión compuesta y en situación de momento tope.

Los resultados son:

$$
\begin{aligned}
q & =0,45 \\
U_{s}^{\prime} & =0,241 \times 180.000=43.380 \mathrm{kp} \\
U_{s} & =(0,45+0,241) \times 180.000-90.000=34.380 \mathrm{kp}
\end{aligned}
$$

La suma de ambas armaduras es:

$$
U_{s}^{\prime}+U_{s}=43.380+34.380=77.760 \mathrm{kp}
$$

mientras que la suma de las armaduras en el ejemplo 9. es:

$$
U^{\prime}{ }^{\prime}+U_{s}=63.000+26.280=89.280 \mathrm{kp}
$$




\section{résumé}

Classification de sections rectangulaires de béton armé pour le critères de calcul: compression, interpolation et flexión (Instruction EH-73)

Julián Navarro Gutiérrez, Dr. architecte

Dans n'importe quel cas de calcul d'une section rectangulaire de béton armé, à partir de ses données et avant de faire le calcul corres. pondant, il est possible de déterminer facilement deux chiffres qui, adoptés comme coordonnées, déterminent un point. La situation de ce point sur son plan de représentation indique le système d'équations qui doit être utilisé pour le calcul de la section. Dans les cas de comptabilité il y a quatre possibilités: compression (simple ou composée), compression interpolée, flexión interpolée et flexion (simple ou composée). A chacune de ces possibilités correspond, sur un plan de représentation, une enceinte déterminée, finie ou infinie. Pour obtenir les contours de cette enceinte il est nécessaire de choisír, préalablement, la valeur du paramètre $\varrho=$ coefficient de recouvrement des armatures. $s_{c}=$ coefficient de rupture du béton.

Presque tous les cas courants de calcul se groupent en:

I. Détermination de sections pour lesquelles la largeur b est une donnée et le coté $d$ une inconnue;

II. Détermination des section pour lesquelles $b$ et $d$ sont des données.

A chaque groupe correspond un graphique déterminé sí, préalablement, ont été établies les valeurs de $\varrho$ et $s_{c}$.

\section{summary}

Classification of reinforced concrete rectangular sections by design criteria of compression, interpolations and deflections (Instruction EH-73)

Julián Navarro Gutiérrez, Dr. in Architecture

In every case of design of a reinforced concrete rectangular section element, it is possible to readilly determine two quantities, which taken as $x-y$ coordinates will define a point, these numbers being taken from the initial information previous to the actual calculations. The position of such point in the plot indicates the equation system to be used in the calculation of the section. When compatibility occurs there are four possibilities: compression (single of multiple), interpolated compression, interpolated deflection and deflection (single or multiple). To each of these possibilities corresponds a given finite or infinite space in the plot. In order to obtain the limits of these spaces, one must first chose the $\varrho=$ ccefficient of the reinforcement covering.

$\mathrm{s}_{\mathrm{c}}=$ concrete ultimate modulus.

Almost all common design and calculation cases are grouped as follows:

I. Determination of a section where the width $b$ is known and the height $d$ is unknown.

II. Determination of sections where both $b$ and $d$ are known.

If the $\varrho$ and $s_{c}$ values have been previously established, each of the above groups will have its own given plotted graph.

\section{zusammenfassung}

Einordnung Rechteckiger Stahlbetonsabschnitte nach Folgenden Berechnungskriterien: Druck, Interpolation und Biegung (Anleitung TH-73)

Dr. Julián Navarro Gutiérrez, Architekt

Bei jeder Berechnung rechteckiger Stahlbetonabschnitte, können auf Grund ihrer Daten und vor der Durchtührung der entsprechenden Berechnung leicht zwei Zahlen bestimmt werden, welche -als Koordnaten- einen Punkt bestimmen. Die Lage dieses Punktes zeigt in seine Darstellungsebene das für die Berechnung des Abschnittes anzuwendende Gleichungssystem an. Bei Verträglichkeit bestehen vier Möglichkeiten: Druck (einfach oder zusammengesetzt), interpolierter Druck, interpolier'e Biegung und Biegung (einfach oder zusammengesetzt). Jeder dieser vier Möglichkeiten entspricht ein bestimmtes Gebiet, sei es begrenzt oder unbegrenzt, auf der Darstellungsebene. Zur Berechnung der Umris se dieser Gebiete ist vorher der Parameter $\varrho=$ Verkleidungskoeffizient der Bewehrungen zu wählen.

$\mathrm{s}_{\mathrm{c}}=$ Beton-Risskoeffizient

zu wählen.

Fast alle normalen Berechnungsfälle können in folgenden Gruppen zusammengefasst werden:

I. Bestimmung der Abschnitte, in denen die Breite b bekannt und die Kante d unbekannt ist,

II. Bestimmung der Abschnitte, in denen $b$ und $d$ bekannt sind.

Wenn im Voraus die Werte für $\varrho$ und $s_{c}$ bestimmt wurden, entspricht jeder Gruppe eine bestimmte graphische Darstellung. 